\title{
RNA amplification and cDNA synthesis for qRT-PCR directly from a single cell
}

\begin{abstract}
Gene expression studies by quantitative reverse-transcription PCR (qRT-PCR) can be challenging when attempted with samples of a few cells or a single cell. Epicentre's MessageBOOSTER ${ }^{\text {TM }}$ CDNA Synthesis from Cell Lysates kit enables RNA amplification and cDNA synthesis directly from cell lysates. A MessageBOOSTER kit reaction from a single cell produces enough cDNA for thousands of qPCRs, even with low-abundance messages.
\end{abstract}

Although qRT-PCR studies provide valuable information about the relative expression of transcripts in a cell, they are difficult to perform with very small numbers of cells (for example, 1-1,000 cells), for a variety of reasons. These include difficulty in purifying extremely small amounts of total RNA, a lack of sensitivity, the need to collect samples often because of the very limited amount of RNA available and the inability to archive samples for future analysis. The new MessageBOOSTER cDNA Synthesis from Cell Lysates kit eliminates these difficulties by enabling RNA amplification and cDNA synthesis directly from cell lysates, without the need to purify total RNA.

Here we demonstrate that a MessageBOOSTER kit reaction produces enough cDNA directly from the lysate of a single cell for thousands of qPCRs, and that the cDNA produced enables sensitive detection of even low-abundance transcripts.

\section{Method overview}

In the MessageBOOSTER cDNA Synthesis from Cell Lysates kit procedure (Fig. 1) cells are counted, collected and pelleted by centrifugation. The pellet is washed, and the cells are lysed by resuspension in QuickExtract ${ }^{\text {TM }}$ RNA Extraction Solution (provided in the kit).

Next, an oligo(dT) primer containing a T7 promoter and MMLV reverse transcriptase are used to synthesize first-strand cDNA from poly $(\mathrm{A})^{+} \mathrm{RNA}$. After second-strand cDNA synthesis, a high-yield in vitro transcription reaction is used to amplify the poly $(\mathrm{A})^{+} \mathrm{RNA}$. The in vitro transcription reaction generates thousands of RNA molecules for every molecule of double-stranded cDNA template and thus serves as a powerful engine for amplifying the poly $(\mathrm{A})^{+} \mathrm{RNA}$. After in vitro transcription, the samples are treated with DNase I to remove the double-stranded cDNA template as well as a large portion of genomic DNA. The amplified RNA is purified using a spin column and then reverse-transcribed, using random primers, into cDNA.

\section{Judith E Meis \& Anupama Khanna}

Epicentre Biotechnologies, Madison, Wisconsin, USA. Correspondence should be addressed to J.E.M. (judy.meis@epibio.com).

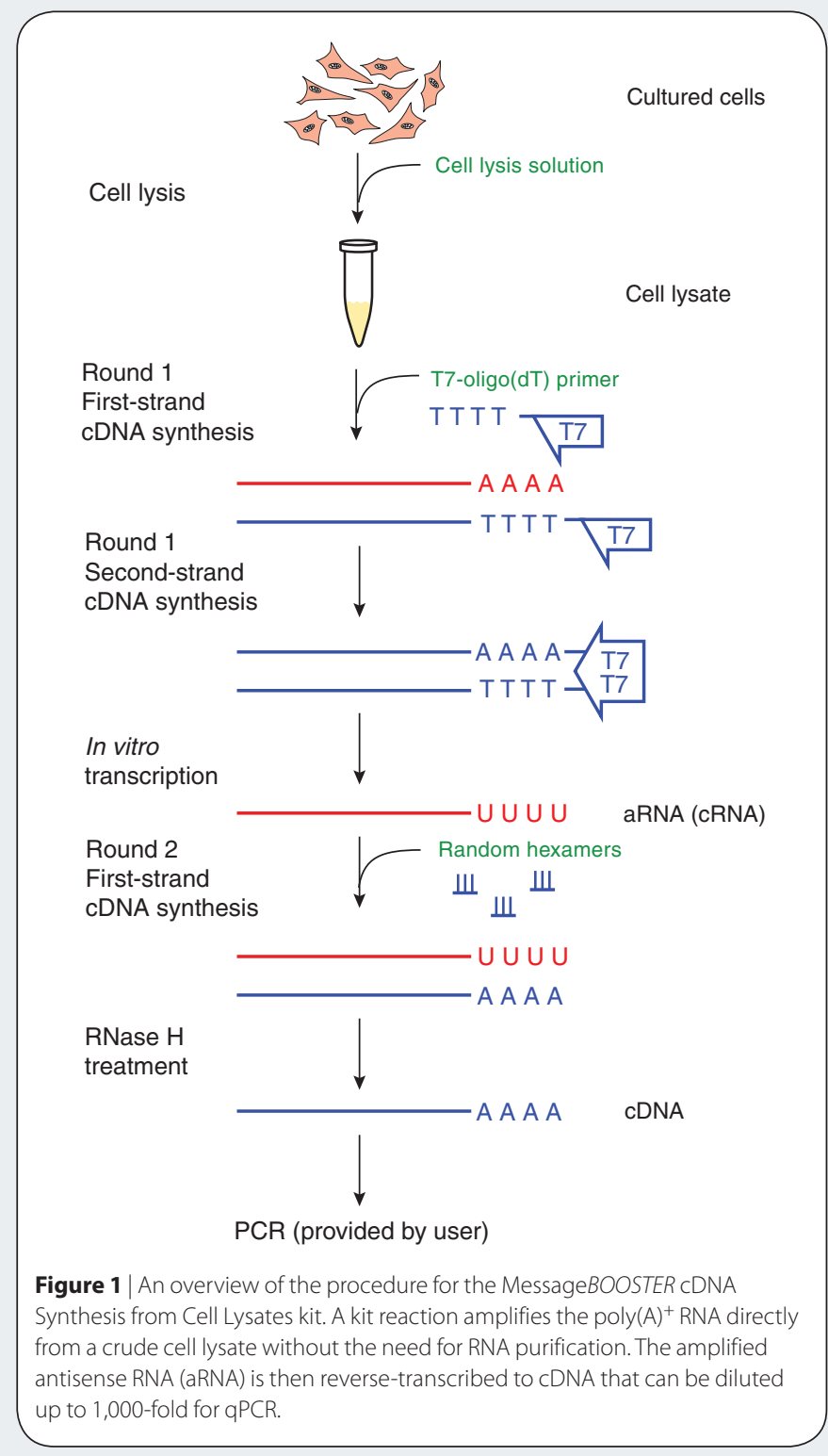



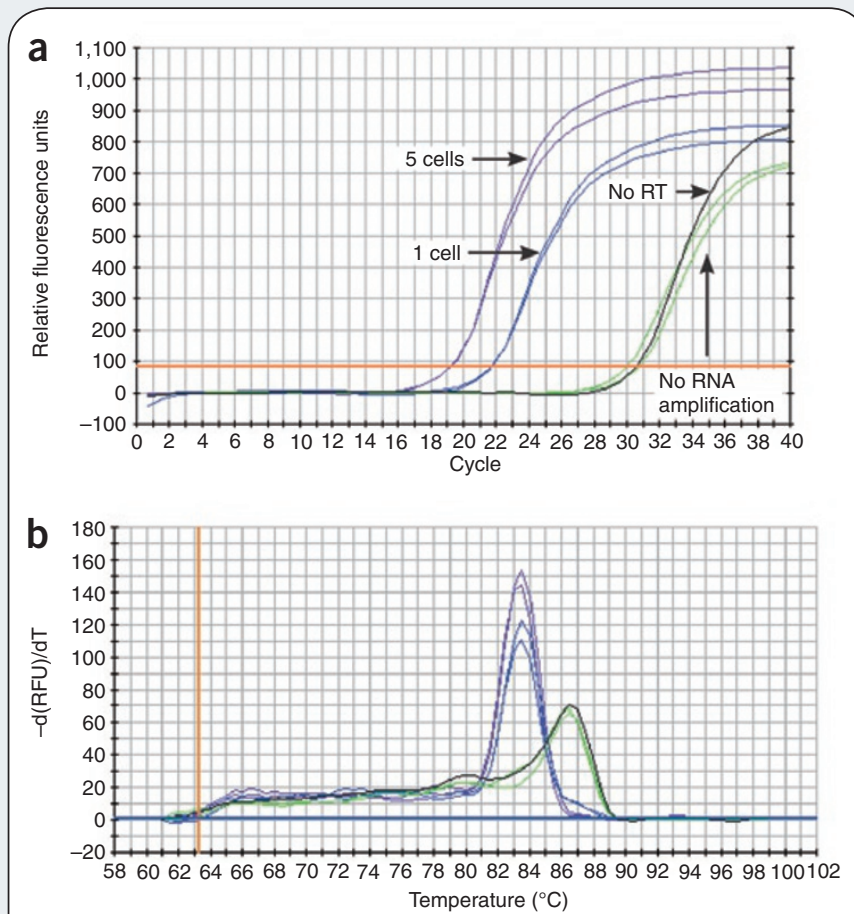

Figure 2 | A MessageBOOSTER kit reaction enables sensitive and specific detection of low-abundance transcripts. (a) qPCR detection of the LDHA transcript in lysates from five HeLa cells and a single HeLa cell using CDNA produced by a MessageBOOSTER kit reaction, by a cDNA-from-cells procedure without benefit of an RNA amplification step, and from a no-RT control reaction All experiments except the no-RT control were performed in duplicate. The horizontal orange line is the fluorescence threshold. (b) Melting-curve analysis of the QPCR products revealed that the MessageBOOSTER CDNA produced an authentic LDHA amplicon, whereas the cDNA-from-cells procedure and no-RT control reaction generated nonspecific amplification products. $T$, temperature; $\mathrm{RFU}$, relative fluorescence units. The vertical orange line represents the temperature at which the cycler begins the melt-curve analysis.

We designate cDNA produced by the MessageBOOSTER kit reaction as 'MessageBOOSTER cDNA'. For control reactions, we synthesized cDNA directly from cell lysates without an RNA amplification step. This cDNA, designated the 'cDNA-from-cells' control, was prepared by reverse transcription using an oligo(dT) primer in a $20-\mu \mathrm{l}$ reaction. In some cases, we used a commercial cDNA-from-cells-type kit. We also performed a MessageBOOSTER kit reaction without the initial reverse transcriptase. This control, designated as the 'no- $\mathrm{RT}^{\prime}$ control, was performed to evaluate the level of residual genomic DNA in the samples after the MessageBOOSTER kit reaction.

Real-time PCR analysis shows benefits of RNA amplification We prepared cDNA from a single HeLa cell using the MessageBOOSTER kit and the cDNA-from-cells procedure (which does not include an RNA amplification step), along with the no-RT control. We performed qPCR with the TAQurate ${ }^{\text {TM }}$ Green Real-Time PCR system (Epicentre) using $1 \mu$ of undiluted cDNA produced by each reaction and a primer pair for the moderately expressed $L D H A$ transcript. The $L D H A$ transcript was readily detected using CDNA produced by a MessageBOOSTER kit reaction from five cells (cycle threshold $(\mathrm{Ct})=19)$ and from a single cell $(\mathrm{Ct}=22)$ (Fig. 2a). The cDNA-from-cells and the no-RT control produced only non-

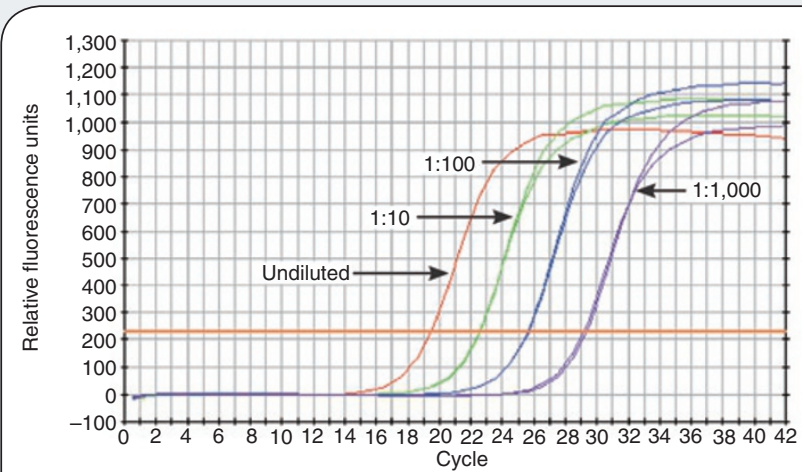

Figure 3 A MessageBOOSTER kit reaction produces sufficient CDNA from a singlecell lysate for at least 1,000 sensitive QPCRs. qPCR was performed using undiluted, 1:10 diluted, 1:100 diluted and 1:1,000 diluted cDNA from a lysate of a single NRK cell. The low-abundance PBGD transcript was readily detected.

specific signals, as determined by melting curve analysis (Fig. $\mathbf{2 b}$ ) and confirmed by gel electrophoresis (data not shown). The melting temperature of the nonspecific products was substantially different from that of the $L D H A$-specific amplicon. Thus, the RNA amplification step, included in the MessageBOOSTER kit reaction, enables sensitive and specific detection of transcripts from a single-cell lysate and reduces the amount of genomic DNA to undetectable levels. In contrast, the cDNA-from-cells procedure yielded cDNA that did not detect the desired transcript in qPCR.

\section{Sensitive detection of low-abundance transcripts}

We also investigated the detection limit of a low-abundance transcript using CDNA produced by a MessageBOOSTER kit reaction from a singlecell lysate. MessageBOOSTER cDNA produced from a single NRK cell lysate was diluted 1:10, 1:100 and 1:1,000 before qPCR analysis. CDNA diluted 1:1,000 enabled detection $(\mathrm{Ct}=29)$ of the low-abundance $P B C D$ transcript (Fig. 3). Melting curve analysis (data not shown) confirmed that the correct amplicon was produced. Thus, a MessageBOOSTER kit reaction enables sensitive detection of a low-abundance transcript using up to a 1,000-fold dilution of the cDNA produced; that is, the kit produces sufficient cDNA for $>1,000$ qPCRs from a single-cell lysate. The number of qPCRs that can be performed using cDNA produced by the MessageBOOSTER kit depends on the number of cells and the abundance of the transcript(s) of interest.

\section{Conclusions}

cDNA produced by a MessageBOOSTER cDNA Synthesis from Cell Lysates kit reaction eliminates the difficulties frequently encountered in qRT-PCR gene expression studies with very small numbers of cells.

First, the kit enables the user to amplify the mRNA and convert it to cDNA directly from a cell lysate, with no loss of RNA.

Second, the linear RNA amplification step improves the sensitivity of detecting even low-abundance transcripts from as little as one cell.

Third, a single MessageBOOSTER kit reaction produces enough cDNA for thousands of qPCRs.

This article was submitted to Nature Methods by a commercial organization and has not been peer reviewed. Nature Methods takes no responsibility for the accuracy or otherwise of the information provided. 